\title{
Taxing Unhealthy Food: Is it an Effective Health Promotion Policy?
}

\author{
Hiba Farhat
}

Pursuing my Master's degree in the department of family medicine, I have the opportunity to work with research experts to study factors affecting health behavior change in the Quebec population. More specifically, my focus is on socio-economic status and the quality of care patients receive as possible predictors of health behavior change over time. Looking through the vast literature on health behaviors and primary prevention, it was unfortunate to see that most behavioral and health promotion studies have not been translated into practice. Additionally, most evidence-based health promotion interventions that have been developed through years of research show little to no effect on long-term outcomes. These findings made me contemplate the extent to which my research results will be disseminated and put into use. This notion pushed me to think of possible ways in which one can build an environment that is supportive of health behavior change. I came to the conclusion that prior to examining the effects of healthcare delivery on health behaviors, it is important to implement healthcare policy changes that encourage patients to adopt and maintain their healthy behaviors.

One of the most effective policies that resulted in a major health behavior change in the past was the taxation of tobacco products. Making tobacco products unaffordable to most people, resulted in a change in the purchasing behavior of Canadians. Additionally, advertisements that promote smoking were banned, which also resulted in a further decrease in the proportion of people that smoke. Building on this notion, the premise underlying this letter is that taxing unhealthy foods can result in better health behaviors in the

*To whom correspondence should be addressed:

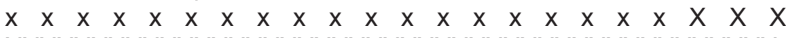
XXXXXXXXXXXXXXXXXXXXXXXXXXXXXXXXXXXXXXXXXXXXXHIBA FARHAT population. Although there are multiple ways in which government policies can promote healthier diets, applying taxes on foods deemed unhealthy has received global attention. In doing so, the government can potentially change the purchasing behavior of the population, which would lead to healthier diets. This has been shown effective especially among children. In a study done by Beydoun et al., they were able to show that slightly increasing the price of fast food resulted in a decrease of total energy intake with an increase in fruits and vegetable intake.1 However, before implementing such policies, all the possible negative implications should be explored and possibly minimized.

One of the main concerns about introducing food taxation is adding financial constraints onto individuals with low income. According to studies done by the Canadian Food Information Council, cost can highly influence the food purchases of those with a household income of less than $\$ 35,000$. Hence, to avoid such inequalities, subsidizing healthy alternatives, in addition to the taxation of unhealthy foods is necessary. Studies have shown that decreasing the price of healthy foods paired with minimal promotion in high schools resulted in an approximate $400 \%$ increase in sales. 2 As a matter of fact, implementing such changes can result in greater health equalities between different socio-economic groups. Unhealthy foods have been shown to be considerably cheaper than healthy alternatives thus low-income individuals are more likely to purchase the former. Hence, lowering this discrepancy in food cost can change the purchasing behavior of the general population, and specifically those with low-income.

This taxation policy seems feasible since revenue generated through taxing unhealthy foods can be utilized to subsidize healthy alternatives. However, some issues and challenges have to be overcome prior to implementation. Firstly, there is a plethora of studies available on food and diet in the 
literature, but there is a debate on what constitutes health and unhealthy foods. Thus, a consensus for the definition of healthy food should be established. Secondly, it is difficult to determine how large the food tax should be to have a significant effect on the population. Hence, further research is necessary to ensure the effectiveness of such a policy.

All in all, taxing unhealthy foods and subsidizing healthy alternatives can provide an encouraging environment for people to adopt healthier lifestyles. Additionally, this health promotion policy can help maintain the effects of other healthcare interventions that will be introduced in the future.

\section{REFERENCES}

1 Beydoun, M. A., Powell, L. M., Chen, X. \& Wang, Y. Food prices are associated with dietary quality, fast food consumption, and body mass index among U.S. children and adolescents. J Nutr 141, 304-311, doi:jn.110.132613 [pii]10.3945/jn.110.132613 (2011).

2 French, S. A. et al. Pricing strategy to promote fruit and vegetable purchase in high school cafeterias. J Am Diet Assoc 97, 1008-1010, doi:S0002-8223(97)00242-3 [pii]10.1016/S0002-8223(97)00242-3 (1997). 